\title{
Japan opens new era in university funding
}

Tokyo. A sweeping reform of the way in which the Japanese government funds research, particularly in universities, has been launched with the introduction of a new grant scheme by an agency affiliated with the Ministry of International Trade and Industry (MITI).

The first grants from MITI's New Energy and Industrial Technology Development Organization (NEDO) were announced last week, with 95 of 109 going to universities. This marks the first time that a government department other than the Ministry of Education, Science, Sports and Culture (Monbusho) has been allowed to award a substantial number of large grants to university researchers (see Nature 376, 110; 1995).

NEDO has won further funds for the scheme, which is open to researchers in universities and national research institutes, in a supplementary budget announced on 20 September, and MITI has moved to institutionalize the scheme in its budget request for next fiscal year (which begins on 1 April 1996). Furthermore, both Monbusho and the Science and Technology Agency (STA), formerly bitter rivals, have announced similar schemes to fund research within each other's domains.

Taken together, the new schemes are the first examples of multiple-source funding across the spectrum of government research; previously, each ministry and agency usually only funded research organizations within their sphere of control. Competition for the first batch of grants, each worth $¥ 50-200$ million (US\$0.5-\$2.0 million), was fierce; applicants outnumbered recipients by a factor of 20 . The grants provide a "very good opportunity for national institutes and university researchers to compete and survive", says one successful applicant, Yoji Mitsui of MITI's National Institute of Bioscience and Human Technology.

The grant approval process involved an assessment by a committee drawn from universities, national institutes and the private sector. The grants are intended to lay the foundations for new industrial technologies in fields such as new materials and biotechnology. Predictably, most of the grants have been awarded to leading national universities, and two-thirds have gone to laboratories in the region centred on Tokyo.

The new funds are expected to be widely welcomed in universities because of the low level of Monbusho's grants. The education ministry's budget for competitive grants for this fiscal year totalled only $¥ 92.4$ billion, most of which is parcelled out in small grants of only a few million yen. The new NEDO awards, totalling $¥ 10$ billion, are therefore a significant boost to university research. Furthermore, each NEDO grant includes money for one or two postdoctoral fellowships, a factor that is missing from most existing Monbusho grants.

The NEDO funds come out of a special supplementary budget prepared earlier this year to cope with the recent earthquake disaster in Kobe. NEDO has won a further $¥ 5$ billion for the scheme in the most recent supplementary budget, and MITI has applied for $¥ 2.65$ billion in next year’s budget for a similar scheme that will run on a regular annual basis.

Until now, Monbusho has been reluctant to allow other ministries and agencies to

\section{Microchip Mark 1 goes up for auction}

\author{
London. A section of computing \\ history was due to go under \\ the hammer this week with \\ the sale of part of Charles \\ Babbage's Difference Engine \\ No.1 (right) at the London \\ auctioneers Christie's. \\ The device is one of six \\ assembled in 1879 from the \\ remaining components of the \\ original Engine by Babbage's \\ son Henry. \\ The section offered for sale, \\ owned by the family of a \\ grandson in New Zealand to \\ which it had been sent, was \\ expected to fetch $£ 50,000$. Babbage \\ was unable to finish the machine, \\ although the Science Museum in \\ London has managed to construct a \\ working model of a revised version of \\ the Engine, using the original designs.
}

provide funds to Japan's universities, over which it has direct control. But a spokesman for the ministry says that it is happy to cooperate with the NEDO scheme, and would have no objection to grants continuing on a regular basis, or to other agencies deciding to award funds to university researchers.

In a remarkable about-turn on previous policy, he says, MITI and Monbusho have "transcended the idea of individual territories" in order to develop Japanese science and technology in the best possible way.

In line with this new mood of cooperation, the STA has announced it will vastly expand its support for university research. Starting with $¥ 5.1$ billion requested in 1995 ’s second supplementary budget, the STA will offer 48 grants, each worth $¥ 1$ billion over five years, to applicants from universities, as well as national research institutes operated by MITI and the STA itself.

This programme will be further expanded with another $¥ 15$ billion requested by the STA for fiscal 1996. This money, which will be part of STA's ordinary annual budget, will be used to fund a further 40 projects.

Decisions on who will receive these grants will be made by a committee formed by the STA, drawing on researchers in universities, national institutes and the private sector. Applications for the first batch of grants are due in November, and successful candidates will be advised in January.

Perhaps the most surprising development of all comes from Monbusho itself. The ministry has asked the government for $¥ 11$ billion in the next fiscal year to provide large grants not only to university researchers but also to researchers in national institutes overseen by MITI and STA.

Akito Arima, former president of Tokyo University and current president of the Institute of Physical and Chemical Research (RIKEN), who has been campaigning for the reform of Japan's university research system, says the new spirit of cooperation between different government agencies is "extremely good" for research in universities and national research institutes, as it opens up multiple sources of funding. But he warns that it may "only stimulate applied science", as disciplines which lack immediate applications, such as astronomy and high-energy physics, will not be covered by the new schemes.

Fumio Kodama of Tokyo University's Research Center for Advanced Science and Technology points out, however, that it would be strange for either MITI or the STA to fund academic science, as these institutions are dedicated to supporting industry-related research. Rather, he suggests that Monbusho should play a "balancing role" by channelling more of its money into purely academic research.

Stephen Barker 